\title{
Estudio de factibilidad para la aplicación de algoritmos de estimación de distribución al problema de secuenciación de vehículos
}

\section{A feasibility study for the application of estimation of distribution algorithms to the car sequencing problem}

\author{
VELÁZQUEZ-JUÁREZ, Juan Carlosł**', PUGA-SOBERANES, Héctor José’, PADIERNA-GARCÍA, \\ Luis Carlos" y SÁNCHEZ-MÁRQUEZ, Elvi Malintzin'
}

Tecnológico Nacional de México/Instituto Tecnológico de León, México.

"Universidad de Guanajuato/División de Ciencias e Ingenierías Campus León, México.

ID $1^{\text {er }}$ Autor: Juan Carlos, Velázquez-Juárez / ORC ID: 0000-0002-7659-6280, CVU CONACYT ID: 930308

ID $1^{\mathrm{er}}$ Coautor: Héctor José, Puga-Soberanes / ORC ID: 0000-0002-1315-1436

ID $2^{\text {do }}$ Coautor: Luis Carlos, Padierna-García / ORC ID: 0000-0002-7474-9159

ID $3^{\text {er }}$ Coautor: Elvi Malintzin, Sánchez-Márquez / ORC ID: 0000-0003-2395-4459

DOI: $10.35429 / J I E .2020 .11 .4 .31 .42$

Recibido Enero 10, 2020; Aceptado Marzo 31, 2020

\section{Resumen}

El principal objetivo de una planta de producción de automóviles es entregar en tiempo y forma los pedidos que se reciben a diario. Estos pedidos no son homogéneos ya que involucran grandes cantidades de autos que generalmente pertenecen a distintos modelos y deben ser pintados con colores diferentes. El problema de secuenciación de vehículos que toma en cuenta estas características fue propuesto por la compañía Renault en 2005 como parte del ROADEF Challenge. Este problema es NP-Duro y se han propuesto diversas técnicas para resolverlo, desde métodos exactos hasta distintos algoritmos heurísticos. En este trabajo se presenta un estudio de factibilidad para aplicar dos Algoritmos de Estimación de Distribución (EDAs) para resolver este problema. Se presentan, además, tres aspectos importantes: el proceso de adaptación de los algoritmos, una técnica para la ejecución de los algoritmos denominada "Enfoque Escalonado con Descarte" y una metodología que implica tolerancia en la sustitución de los individuos. También se muestran los resultados obtenidos por los algoritmos. El análisis de resultados muestra el proceso de la adaptación de los algoritmos al problema y los ajustes que pueden hacerse para mejorar su competencia respecto al estado del arte.

Problema de Secuenciación de Vehículos, Algoritmos de Estimación de Distribución, ROADEF Challenge

\begin{abstract}
The main objective of an automobile production plant is to deliver on time and form the orders that are received daily. These orders are not homogeneous since they involve large quantities of cars that generally belong to different models and must be painted in different colors. The car sequencing problem that takes these characteristics into account was proposed by the Renault Company in 2005 as part of the ROADEF Challenge. This problem is NP-Hard and various techniques have been proposed to solve it, from exact methods to different heuristic algorithms. This work presents a feasibility study to apply two Distribution Estimation Algorithms (EDAs) to solve this problem. In addition, three important aspects are presented: the adaptation process of the algorithms, a technique for the execution of the algorithms called the "Stepped Approach with Discard" and a methodology that involves tolerance in the substitution of the individuals. The results obtained by the algorithms are also shown. The analysis of the results shows the algorithms adaptation process and the adjustments that can be made to improve their competence with the state of art.
\end{abstract}

Citación: VELÁZQUEZ-JUÁREZ, Juan Carlos, PUGA-SOBERANES, Héctor José, PADIERNA-GARCÍA, Luis Carlos y SÁNCHEZ-MÁRQUEZ, Elvi Malintzin. Estudio de factibilidad para la aplicación de algoritmos de estimación de distribución al problema de secuenciación de vehículos. Revista de Ingeniería Industrial. 2020. 4-11:31-42.

\footnotetext{
*Correspondencia al Autor (Correo Electrónico: velazquezjuarezjc@ @otmail.com)

$\dagger$ Investigador contribuyendo como primer autor.
} 


\section{Introducción}

El proceso de manufactura de automóviles suele llevarse a cabo en tres talleres: el taller de carrocería donde se prepara el esqueleto de los vehículos, el taller de pintura que es el encargado de pintar los autos según los pedidos recibidos y la línea de ensamble donde se instalan todos los componentes que debe tener cada modelo de automóvil. En 2005 se llevó a cabo el ROADEF Challenge organizado por la Asociación Francesa de Investigación de Operaciones y Análisis de Decisión cuyo tema principal fue una variante del problema de secuenciación de vehículos (CSP por sus siglas en inglés) presentado por la empresa manufacturera Renault.

El principal objetivo de este desafío fue resolver una versión multiobjetivo del CSP clásico que toma en cuenta los talleres de pintura y la línea de ensamble. Varios equipos alrededor del mundo participaron en este desafío utilizando diversas técnicas de optimización, desde métodos exactos como Programación de Restricciones o Programación Entera hasta métodos heurísticos como Búsqueda local u Optimización por Colonia de Hormigas, entre otros. Una reseña del desafío puede encontrarse en (Solnon, Cung, Nguyen, \& Artigues, 2008). Desde entonces, se han desarrollado mejoras a los métodos utilizados o se han propuesto nuevas aproximaciones. En este trabajo se proponen dos métodos pertenecientes a la familia de los Algoritmos de Estimación de Distribución para estudiar su factibilidad.

Los algoritmos propuestos han demostrado ser una herramienta poderosa en la solución de distintos problemas de optimización debido a que utilizan la información proveniente de los individuos generados para estimar su distribución estadística. Una vez obtenida esta información, construyen un modelo probabilístico a partir del cual se crean nuevos individuos de manera iterada.

Los métodos a implementar son: una versión discreta del Algoritmo de Estimación de Distribución Marginal Univariante de Boltzmann (BUMDA) (Valdez-Peña, Hernández, \& Botello, 2013) y el Algoritmo de Estimación de Distribución Multinomial (MEDAL) (Duarte-Carrera, Rojas-Domínguez, \& Padierna García, 2018).
Estos

algoritmos

presentan

características comunes y algunas diferencias que los hacen obtener algunas ventajas al dar solución al problema. Por una parte, BUMDA calcula los parámetros necesarios (i.e. media y varianza) para generar una distribución normal utilizada para aproximarse a la distribución de Boltzmann. Después de calcular esta distribución, se hace un muestreo aleatorio para generar nuevos individuos. MEDAL calcula las frecuencias de los componentes en los mejores individuos de la población, con estas frecuencias se construye una distribución multinomial y, mediante un muestreo aleatorio, se genera una nueva población de individuos. Además, se presenta una técnica para la ejecución de los algoritmos denominada "Enfoque Escalonado con Descarte" que es una mejora a las aproximaciones utilizadas en la literatura para abordar problemas multiobjetivo (Al Chami, Manier, \& Manier, 2019; Palacio \& Larrea, 2017). Esta variante provee a los algoritmos una forma de conservar a los mejores individuos en cada generación.

La siguiente sección describe el Problema de Secuenciación de Vehículos. La sección 3 presenta los algoritmos utilizados y detalla la forma en que se adaptaron al CSP. En la sección 4 se muestra cómo fueron realizados los experimentos mientras que en la sección 5 se puntualizan los resultados obtenidos y su análisis. Por último, la sección 6 presenta las conclusiones de este trabajo.

\section{Descripción del problema}

La fabricación de automóviles se caracteriza por el hecho de que los autos producidos en un solo día en una línea de ensamblaje sencilla no son homogéneos: cada auto puede requerir un conjunto de opciones ligeramente diferente (Parrello, Kabat, \& Wos, 1986). El objetivo del problema de secuenciación de vehículos es minimizar el número total de sobrecargas en las estaciones de trabajo, estas estaciones son las encargadas de instalar las opciones (también conocidas como componentes) requeridas por cada auto, este objetivo también es conocido como suavizado de restricciones. Una secuencia puede definirse como una permutación de todo el conjunto de automóviles que deben ser producidos, mientras que una subsecuencia es un elemento de alguna partición de la secuencia. 
Una sobrecarga se produce cuando la cantidad de autos que requieren una opción es mayor a la capacidad que tiene la estación encargada de instalar dicho componente. Este problema fue descrito por Parello en 1986 como una variante del Job-Shop Scheduling Problem (JSSP), donde cada trabajo es representado como un auto que requiere un conjunto de opciones a ser instaladas (aire acondicionado, estéreo, quemacocos, etc.) el cual debe secuenciarse en una línea de ensamblaje. Un auto puede requerir un conjunto ligeramente distinto de opciones que su predecesor o su sucesor y cada estación de trabajo (donde se instalan dichas opciones) tiene una restricción de capacidad $p_{i} / q_{i}$. Esta restricción indica que, para cualquier subsecuencia de autos consecutivos de tamaño $q_{i}$, a lo mucho $p_{i}$ de ellos pueden requerir la opción que se instale en esa estación de trabajo. Distintos autores (Estellon \& Gardi, 2013; Ian P., 1998; Kis, 2004) han demostrado que este problema es NP-Duro. Originalmente, el CSP fue presentado como un problema de satisfacción de restricciones que solo incluía el objetivo de suavizado de restricciones, aunque también puede definirse como un problema de optimización combinatoria debido a que las soluciones son representadas como vectores con permutaciones de números enteros. Estos números representan los índices que se le asigna a cada auto dentro del conjunto de autos a secuenciar. En este trabajo, el CSP es abordado como un problema de optimización combinatoria.

De acuerdo con (Solnon, Cung, Nguyen, \& Artigues, 2008), una instancia del problema de CSP clásico se define por una tupla $(V, O, p, q, r)$ donde:

- $\quad V=\left\{v_{1}, \ldots, v_{n}\right\}$ es el conjunto de autos a ser producido.

$O=\left\{o_{1}, \ldots, o_{m}\right\}$ es el conjunto de opciones.

$p: O \rightarrow N$ y $q: O \rightarrow N$ definen la restricción de capacidad para cada opción $o_{i} \in O$. Esta restricción establece que, para cada subsecuencia de $q_{i}$ autos consecutivos en la línea de ensamblaje, como máximo $p_{i}$ autos deben requerir $o_{i}$.
- $r: V \times O \rightarrow\{0,1\}$ definen los requerimientos de las opciones, es decir, para cada vehículo $v_{j} \in V$ y para cada opción $o_{i} \in O$, se tiene que:

$r_{j i}=\left\{\begin{array}{cc}1 & \text { si } o_{i} \text { debe instalarse en } v_{j} \\ 0 & \text { en otro caso }\end{array}\right.$

El espacio de búsqueda para una instancia del CSP está compuesto por todas las posibles permutaciones de los autos de $V$.

En 2005, la compañía RENAULT propuso una variante del CSP para el ROADEF Challenge donde divide el objetivo de suavizado en dos: uno que toma en cuenta las estaciones de alta prioridad y el otro las estaciones de baja prioridad. Además, se añade un tercer objetivo que involucra restricciones de pintura por lotes. Este último se debe a que se quiere minimizar el uso del solvente que se utiliza al limpiar el equipo encargado de pintar los autos. Con estas modificaciones y conforme a (Solnon, Cung, Nguyen, \& Artigues, 2008), se puede definir al CSP propuesto por RENAULT como:

- Una instancia del CSP clásico como se definió anteriormente.

Dos subconjuntos $O_{H}$ y $O_{L}$ de $O$ donde $O_{H}$ contiene el conjunto de opciones de alta prioridad y $O_{L}$ contiene el conjunto de opciones de baja prioridad. Estas opciones son instaladas en las estaciones de alta y baja prioridad mencionadas anteriormente.

- Un conjunto de colores $C$ y una función $c$ : $V \rightarrow C$ que asocia con cada vehículo $v_{i} \in$ $V$ con un color $c_{i}$.

Un límite de autos del mismo color que pueden secuenciarse consecutivamente. Este límite se representa con $B \in N$.

- Una secuencia que contiene los últimos vehículos secuenciados pertenecientes al día anterior. Esto permite tomar en cuenta el día de producción anterior cuando se calcula el número de sobrecargas y cambios de color. 
El CSP propuesto por RENAULT se plantea como un problema de optimización multiobjetivo que puede ser resuelto de forma lexicográfica asignando pesos a cada uno de los objetivos dependiendo de su nivel de prioridad. La evaluación de una solución se lleva a cabo mediante la suma de cada valor fitness de los tres objetivos multiplicado por su respectivo peso. En la ecuación (1) FT representa el fitness total obtenido por la suma lexicográfica de $F_{1}, F_{2}$ y $F_{3}$ que pueden tomar el valor de cualquiera de los tres objetivos: $F H$ para las estaciones de alta prioridad, $F L$ para las estaciones de baja prioridad y $F C$ para los cambios de color dependiendo de la instancia. $\alpha, \beta$ y $\gamma$ son los pesos que se asignan a cada objetivo de tal manera que $(\alpha<\beta<\gamma)$.

$$
F T=\alpha * F_{1}+\beta * F_{2}+\gamma * F_{3}
$$

En este trabajo de investigación se utilizó la expresión (2) en la que no se privilegia a ninguno de los objetivos individuales. La finalidad es observar el comportamiento global de los algoritmos propuestos tomando los valores directos de los objetivos individuales.

$$
F T=F_{1}+F_{2}+F_{3}
$$

Existen diversas variantes del método utilizado para evaluar la función objetivo (comúnmente llamada "ventana deslizante") que permite calcular el número de sobrecargas que genera una secuencia dada (véase (Solnon, Cung, Nguyen, \& Artigues, 2008; Fliedner \& Boysen, 2008; Jahren \& Achá, 2018)). Para este trabajo se utilizará la función objetivo como se muestra en la ecuación (3).

$$
F E_{i}(S)=\sum_{a}^{b} F E_{i}\left(S, W_{P_{i}}\right)+\sum_{k}^{\text {NewP }_{i}} F E_{i}\left(S, W_{\text {NewP }_{i}}\right)
$$

Donde $\boldsymbol{F E}_{\boldsymbol{i}}(\boldsymbol{S})$ representa la función fitness de una secuencia $\boldsymbol{S}$ en la $i$-ésima estación de trabajo $\boldsymbol{E}_{\boldsymbol{i}}$. Como se mencionó anteriormente, estas estaciones están divididas en dos categorías: $\boldsymbol{H}$ para las estaciones de alta prioridad y $\boldsymbol{L}$ para las de baja prioridad, de tal manera que $\boldsymbol{E}_{\boldsymbol{i}}$ puede ser sustituida por $\boldsymbol{H}$ o $\boldsymbol{L}$ según sea el caso.
Las variables $\boldsymbol{W}_{\boldsymbol{P}_{\boldsymbol{i}}}$ y $\boldsymbol{W}_{\boldsymbol{N} \text { ew } \boldsymbol{P}_{\boldsymbol{i}}}$ representan las ventanas de la $i$-ésima estación de tamaño $\boldsymbol{P}_{\boldsymbol{i}}$ y $\boldsymbol{N} \boldsymbol{e} \boldsymbol{w}_{\boldsymbol{P}_{\boldsymbol{i}}}$ respectivamente, que se deslizarán a lo largo de la solución contando las sobrecargas ocurridas en la subsecuencia que abarca dicha ventana. En esta versión del CSP, se toman en cuenta los autos que fueron secuenciados en el día de producción anterior pero que no alcanzaron a ser producidos. Para contar las sobrecargas de la secuencia del día actual, se toma en cuenta un subconjunto de los autos del día anterior que quedaron pendientes de procesar. Este subconjunto varía en tamaño ya que depende del índice $\boldsymbol{q}_{\boldsymbol{i}}$ de la estación de trabajo que se está evaluando. Para mayor profundización en la función objetivo, el lector puede referirse a (Sánchez Márquez, y otros, 2019).

El objetivo de cambios de color determina que en una secuencia no puede haber más de $\boldsymbol{B}$ autos consecutivos que requieran ser pintados con el mismo color. Además, este objetivo toma en cuenta las veces que se cambia el color de la pintura. La función que contabiliza los cambios de color en una secuencia $\boldsymbol{S}$ (ecuación (4)) puede definirse como la suma de las diferencias de color $(\boldsymbol{D C})$ entre los autos $\boldsymbol{k}$ y $\boldsymbol{k}+\boldsymbol{1}$ de tal manera que $\boldsymbol{D} \boldsymbol{C}_{\boldsymbol{k}, \boldsymbol{k}+\mathbf{1}}=\mathbf{1}$ si los autos $\boldsymbol{k}$ y $\boldsymbol{k}+\mathbf{1}$ tienen un color diferente y $\boldsymbol{D} \boldsymbol{C}_{\boldsymbol{k}, \boldsymbol{k}+\mathbf{1}}=\mathbf{0}$ en otro caso. Concretamente el objetivo de cambios de color $(\boldsymbol{F C})$ se define como (Chutima \& Olarnviwatchai, 2018):

$$
F C(S)=\sum_{k=0}^{n} D C_{k, k+1}
$$

A diferencia de los otros dos objetivos donde el número de autos tomados del día anterior depende de la restricción de capacidad de la estación de trabajo que se está evaluando, en la ecuación (4) el índice $k$ comienza en 0 ya que se considera al último auto secuenciado el día anterior. Esto se debe a que, si el color del primer auto de la secuencia es distinto al último auto del día anterior, se realiza un cambio de color.

\section{Algoritmos de Estimación de Distribución}

Los Algoritmos de Estimación de Distribución (EDAs) son técnicas de optimización estocástica que exploran el espacio de soluciones potenciales al construir modelos probabilísticos explícitos de las soluciones más prometedoras (Armazañas, y otros, 2011). 
Una de las principales diferencias entre EDAs y los algoritmos evolutivos como los Algoritmos Genéticos (AG), es la forma en que se regenera la población. Los AG utilizan un subconjunto de los individuos más aptos de la población actual mientras que los EDAs emplean una distribución de probabilidad para generar nuevas soluciones.

Esta estrategia permite a los EDAs incrementar la probabilidad de generar la solución óptima en el espacio de búsqueda (Valdez-Peña, Hernández, \& Botello, 2013). El proceso fundamental de cualquier EDA se describe en el Algoritmo 1 (Armazañas, y otros, 2011).

Generalmente, los EDAs trabajan con una población de posibles soluciones al problema, la cual se crea a partir de la distribución uniforme de todas las soluciones admisibles. Después, cada individuo se califica de acuerdo con una función fitness. Esta función evalúa que tan buena es una solución de acuerdo con el tipo de problema (maximización o minimización) y a sus requisitos: por ejemplo, el número de sobrecargas que genera la secuencia de autos creada por el algoritmo.

Una vez obtenidos los valores fitness de cada individuo, la población se somete a un método de selección para elegir a las soluciones más prometedoras con las cuales se construye el modelo probabilístico que intenta estimar la distribución de probabilidad de los individuos seleccionados. Existen diversos métodos para seleccionar a los mejores individuos de una población.

Entre los más comunes destacan aquellos que ordenan a los individuos de acuerdo a su fitness. Sin embargo, existen otros métodos que ayudan a evitar la convergencia prematura que causa el uso de métodos basados en fitness (Gaudín, Madruga, \& Rodríguez, 2020).

Una vez que el modelo es construido, se generan nuevos individuos al realizar un muestreo de la distribución calculada por el modelo. Estas nuevas soluciones se añaden a la vieja población o la reemplazan por completo. Este proceso se repite hasta que se cumple un criterio de paro.
1. $\mathrm{g} \leftarrow 0$

2. generar la población inicial $\mathrm{P}(0)$

3. mientras no se alcance el criterio de paro

3.1. seleccionar los mejores individuos $\mathrm{S}(\mathrm{g})$ de $\mathrm{P}(\mathrm{g})$

3.2. construir un modelo probabilístico de $\mathrm{S}(\mathrm{g})$

3.3. generar nuevos individuos $\mathrm{O}(\mathrm{g})$ con el modelo $\mathrm{S}(\mathrm{g})$ generado.

3.4. incorporar la nueva población $\mathrm{O}(\mathrm{g})$ a la actual $\mathrm{P}(\mathrm{g})$

3.5. $g \leftarrow g+1$

fin mientras

Algoritmo 1 EDA básico

Fuente: (Armazañas, y otros, 2011) [Microsoft Word]

En la última década y media, los EDAs han sido aplicados a muchos problemas de optimización desafiantes (Larrañaga \& Lozano, 2002) tales como diseño de configuración del sistema celular CDMA (Sun, Zhang, Li, \& Yao, 2008), predicción de estructura proteica (Bacardit, y otros, 2007), selección de Peakbin en espectrometría de masas (Armazañas, y otros, 2011), entre otros y aunque en (Ceberio, Irurozki, Mendiburu, \& Lozano, 2012) se señala que varios trabajos encontrados en la literatura confirman el buen funcionamiento de los EDAs aplicados a problemas reales de distintos campos de investigación, tales como problemas de ruteo de vehículos, calibración de aplicaciones químicas, encontrar la ruta optima en espacios 3D, testeo de software, optimización del tratamiento de quimioterapia para cáncer, etc., el autor hace mayor hincapié en los problemas basados en permutaciones, específicamente en 4: el problema del agente viajero (TSP), flow shop scheduling problem (FSSP), el problema de asignación cuadrática (QAP) y el problema de ordenamiento lineal (LOP) indicando que los EDAs también son efectivos en la solución de los problemas de optimización combinatoria.

Esta es una de las principales razones de implementar EDAs a un problema como el CSP ya que, como se explica en la sección 2 , este problema puede ser tratado como un problema basado en permutaciones, además de ser una variante del FSSP. Con esto en mente, en las secciones siguientes se describen los algoritmos utilizados en esta investigación detallando la forma en que fueron adaptados, la representación de los individuos y sus diferencias y similitudes. 


\section{Boltzmann Univariate Marginal Distribution Algorithm (BUMDA)}

BUMDA es un algoritmo de estimación de distribución que utiliza un modelo normal gaussiano para aproximarse a la distribución de Boltzmann. Esta aproximación se logra calculando los parámetros de dicho modelo (media y varianza) con fórmulas derivadas de una minimización analítica de la divergencia Kullback-Leibler. Este algoritmo ha sido utilizado para resolver problemas de optimización con dominio continuo como las funciones Rosenbrock, Sum Cancellation, Ackley, etc. También ha sido aplicado en la optimización de hiper parámetros de los kernels utilizados en máquinas de soporte vectorial. En ambos casos, BUMDA demuestra un buen desempeño comparado con otros EDAs, esto se debe a que emplea selección por truncamiento para dirigir la búsqueda a las regiones más prometedoras en el espacio de búsqueda. En el Algoritmo 2 se detallan los pasos seguidos en BUMDA.

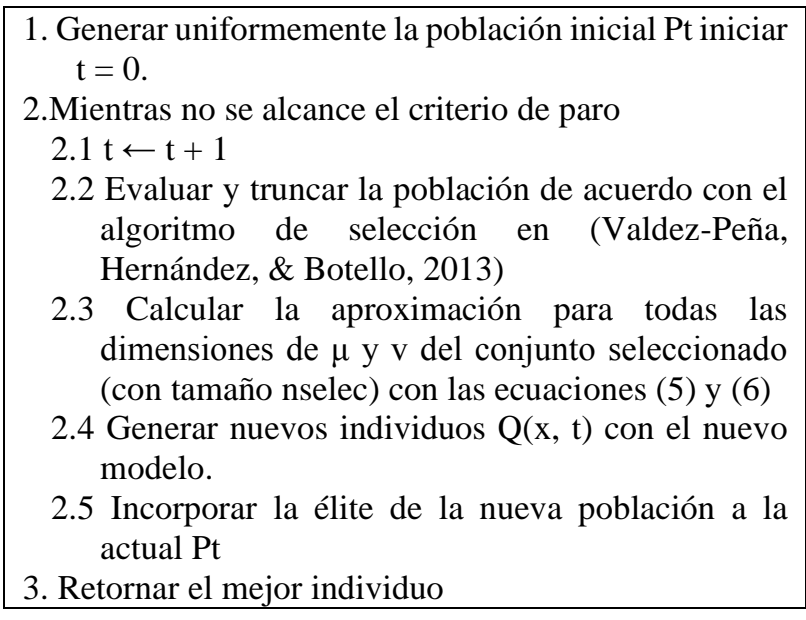

\section{Algoritmo 2 BUMDA}

Fuente: (Valdez-Peña, Hernández, \& Botello, 2013) [Microsoft Word]

Los parámetros que se utilizan en BUMDA son calculados mediante las ecuaciones (5) y (6) donde $\bar{F}\left(x_{i}\right)=F(x)-$ $F\left(x_{\text {selec }}\right)+1$ y $F\left(x_{i}\right)$ es el valor fitness del individuo $x_{i}$.

$$
\begin{aligned}
& \mu \approx \frac{\sum_{1}^{n s e l e c} x_{i} \bar{F}\left(x_{i}\right)}{\sum_{1}^{n \operatorname{selec}} \bar{F}\left(x_{i}\right)} \\
& v \approx \frac{\sum_{1}^{n \operatorname{selec}} \bar{F}\left(x_{i}\right)\left(x_{i}-\mu\right)^{2}}{\sum_{1}^{n \operatorname{selec}} \bar{F}\left(x_{i}\right)}
\end{aligned}
$$

Originalmente en BUMDA, un individuo I es representado como un vector de tamaño $n$ donde cada componente $I \_k \in R$. Debido a que una solución para el CSP es un vector que contiene los índices de los autos a secuenciar y estos índices son números enteros, esta representación no es la adecuada para que la versión original de este algoritmo sea utilizada.

\begin{tabular}{|c|c|c|c|c|c|c|c|c|}
\hline Secuencia & 1 & 2 & 3 & 4 & $\ldots$ & n - 2 & n - 1 & n \\
\hline $\begin{array}{c}\text { Cromosoma } \\
\text { Clase }\end{array}$ & 3 & 2 & 5 & 1 & $\ldots$ & 3 & 3 & 1 \\
\hline $\begin{array}{c}\text { Cromosoma } \\
\text { Color }\end{array}$ & 3 & 3 & 4 & 1 & $\ldots$ & 2 & 2 & 1 \\
\hline
\end{tabular}

Figura 1 Representación de los individuos en BUMDA Fuente: elaboración propia [Microsoft Word]

Por tal motivo, en este trabajo se propone una versión discreta de BUMDA denominada BUMDAD. Esta versión utiliza un par de cromosomas (Chutima \& Olarnviwatchai, 2018) como se muestra en la Figura 1. El primer cromosoma consiste en la clase del auto. El segundo cromosoma representa el color de cada auto. En esta figura, Secuencia representa el índice (posición) que tiene el auto en la instancia original.

Con la información de los cromosomas, se generan dos distribuciones de probabilidad, una para el vector de clase y otra para el vector de color. Para ello se calculan cuatro parámetros denominados $\mu_{\text {clase }}, \mu_{\text {color }}, v_{\text {clase }}$ y $v_{\text {color }}$. Con las distribuciones generadas se realiza un muestreo por cada $k$-ésimo componente generando valores de clase y color para un individuo.

Experimentos preliminares demostraron que este proceso puede generar individuos infactibles, es decir, secuencias con valores fuera del rango de cada vector lo que resulta en autos inexistentes en la secuencia original y/o autos repetidos. Esto es un inconveniente ya que ambos casos implican que uno o varios autos no sean tomados en cuenta al momento de evaluar la secuencia produciéndose un cálculo incorrecto del fitness de estos individuos.

Para preservar la factibilidad de los individuos generados, se utiliza un reparador cromosómico como se especifica en (Sánchez Márquez, y otros, 2019). 
Este reparador, dotado con los rangos de clases y colores correctos, recorre toda la secuencia en busca de valores anormales, una vez detectados son reemplazados con algunos de los valores faltantes considerando también que no se sobrepase el número de autos de cualquier clase o color.

\section{Multinomial Estimation of Distribution Algorithm (MEDAL)}

MEDAL es un algoritmo de estimación de distribución basado en la distribución multinomial aplicable a problemas de optimización discretos. Fue empleado exitosamente para resolver el problema de desenvolvimiento de fase para reconstrucción de objetos 3D en (Duarte-Carrera, RojasDomínguez, \& Padierna García, 2018). El proceso general de MEDAL se describe en el Algoritmo 3.

1. Inicializar aleatoriamente la población de soluciones candidatas

2. Mientras no se alcance el criterio de paro

2.1. Evaluar cada solución conforme la función fitness

2.2. Elegir los mejores $M$ individuos de la población $(M<N)$

2.3. Estimar una distribución multinomial de los $M$ individuos seleccionados

2.4. Generar una nueva población utilizando la distribución multinomial

3. Retornar la mejor solución encontrada

\section{Algoritmo 3 MEDAL}

Fuente: (Duarte-Carrera, Rojas-Domínguez, \& Padierna García, 2018) [Microsoft Word]

Debido a su naturaleza, MEDAL puede clasificarse como un algoritmo orientado a permutaciones. Por lo tanto, se utiliza una estructura más simple que en BUMDA. Esta estructura (Figura 2) está compuesta por un solo vector donde cada componente es el índice de los autos en la secuencia original.

\begin{tabular}{|l|l|l|l|l|l|l|l|l|}
\hline Secuencia & 1 & 2 & 3 & 4 & $\ldots$ & $\mathrm{n}-2$ & $\mathrm{n}-1$ & $\mathrm{n}$ \\
\hline
\end{tabular}

Figura 2 Estructura de un individuo en MEDAL Fuente: elaboración propia [Microsoft Word]

Para estimar la distribución, se selecciona un subconjunto de los mejores individuos de la población. A partir de este subconjunto se construye una matriz de frecuencias que contabiliza las veces que cada auto aparece en cada posición en los individuos seleccionados.
Una vez terminado este proceso, a cada auto se le asigna una probabilidad de ser seleccionado en una posición específica mediante un proceso de selección por ruleta. $\mathrm{Si}$ la frecuencia de determinado auto en cierta posición disminuye, significa que ese auto puede ser recolocado en la secuencia en una posición donde mejore el fitness de los individuos.

Suponiendo un conjunto de 25 autos se obtiene el vector de frecuencias del auto $1 \mathrm{y}$, como se puede observar en la Figura 3, este auto aparece con mayor frecuencia en la posición 12. También destaca el hecho que en las posiciones 19 y 24 la frecuencia es igual a 0 lo que significa que de los $\mathrm{M}$ individuos seleccionados para calcular las frecuencias, en ningún individuo aparece el auto 1 en dichas posiciones.

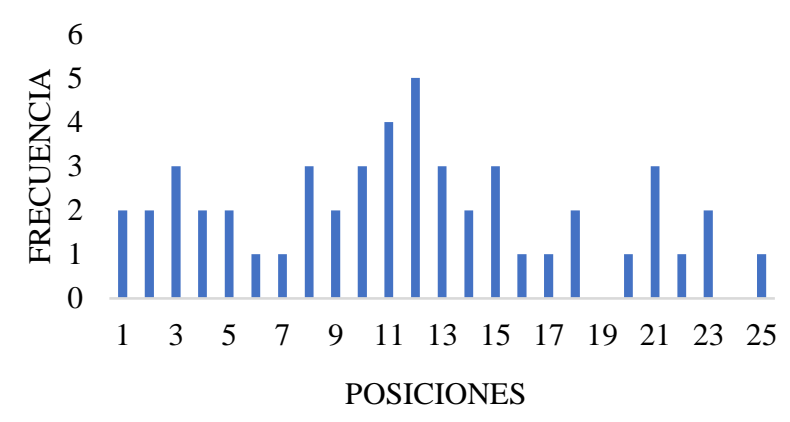

Figura 3 Histograma de frecuencias del auto 1 Fuente: elaboración propia [Microsoft Excel]

La Figura 4 muestra la probabilidad que tiene el auto 1 en ser elegido para cualquiera de las 25 posiciones. Este algoritmo, a diferencia de BUMDA, no hace uso del reparador cromosómico ya que su metodología permite crear individuos factibles en cada iteración.

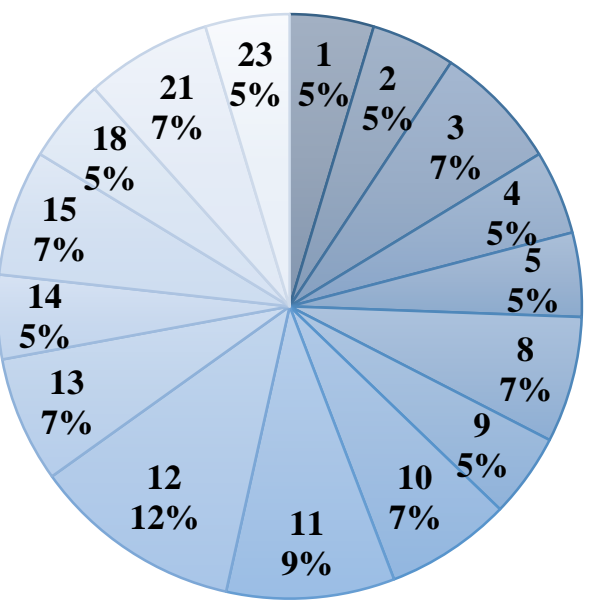

Figura 4 Ruleta de porcentajes del auto 1

NOTA: se omiten los porcentajes menores al $2 \%$ por fines ilustrativos

Fuente: elaboración propia [Microsoft Excel]

VELÁZQUEZ-JUÁREZ, Juan Carlos, PUGA-SOBERANES, Héctor José, PADIERNA-GARCÍA, Luis Carlos y SÁNCHEZ-MÁRQUEZ, Elvi Malintzin. Estudio de factibilidad para la aplicación de algoritmos de estimación de distribución al problema de secuenciación de vehículos. Revista de Ingeniería Industrial. 2020 


\section{Adaptación de MEDAL a CSP con Escalonamiento y Tolerancia}

En esta sección se presentan dos estrategias de adaptación de MEDAL para resolver el CSP. La primera es utilizar un enfoque escalonado con descarte (MEDAL ST) y la segunda es mejorar este enfoque con un método de sustitución de individuos con tolerancia en el proceso de descarte definido como MEDAL CT. Las siguientes subsecciones puntualizan los detalles de estas dos metodologías.

\section{Enfoque Escalonado con Descarte}

El Enfoque Escalonado con Descarte (Sánchez Márquez, y otros, 2019) (Figura 5) consiste en evaluar los objetivos de manera individual respetando en que se presentan en el escalonamiento. Además, este enfoque está orientado a evitar un decremento en los fitness ya calculados cuando se evalúa el siguiente. Lo anterior se logra reutilizando la población final del primer objetivo para ser la inicial del segundo y lo mismo con el tercer objetivo resultando en un conjunto de secuencias evaluadas en cada uno de los objetivos.

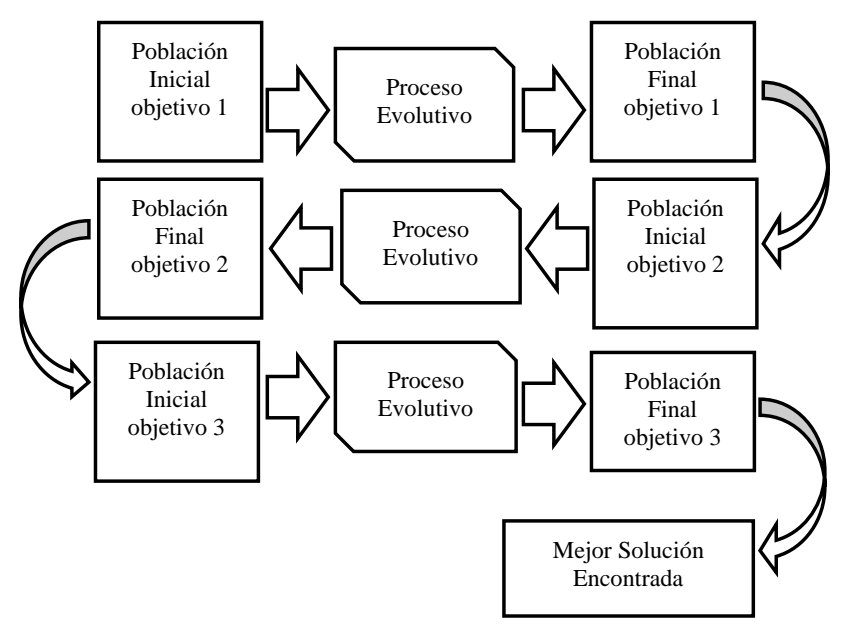

Figura 5 Proceso del Enfoque Escalonado con Descarte Fuente: elaboración propia [Microsoft Power Point]

Durante el proceso evolutivo, se realiza una comparación y sustitución de secuencias. En la comparación se descarta la secuencia que genere un número mayor de sobrecargas (o cambios de color). Cuando se está evaluando el segundo o tercer objetivo, se considera que las secuencias no deben incrementar las violaciones de los objetivos ya evaluados. Si esto sucede, la secuencia es descartada.
Pruebas preliminares demostraron que, con este método, algunas buenas secuencias son descartadas, aunque obtengan un mejor fitness en dos de los tres objetivos. Por esta razón, se propone dar un nivel de tolerancia en la comparación de las soluciones.

\section{Enfoque Escalonado con Tolerancia en el Descarte}

Suponiendo un individuo I en cierta generación gen $_{i}$ tiene un fitness en FH de 10 y en FC su fitness es 20 al transcurso de una generación un nuevo individuo obtiene un fitness de 15 en FC pero en $\mathrm{FH}$ su fitness aumenta una unidad, el enfoque ilustrado en la Figura 5 descarta a este nuevo individuo aunque haya disminuido el FC en 5 unidades. El método de sustitución con tolerancia (CT) permite al algoritmo elegir a este nuevo individuo ya que la ganancia en FC es mayor al aumento del fitness en FH. Para tomar esta decisión se debe fijar un nivel de tolerancia máximo, este nivel se representa como $\theta$, cuando se comparan ambos individuos se calcula la diferencia entre ambos fitness, si esta diferencia es negativa significa que el nuevo individuo es peor que el anterior y se descarta. Si la diferencia es positiva el nuevo individuo es mejor que el anterior y se procede a comparar los fitness del objetivo anterior. Se calcula la diferencia entre estos objetivos y si la diferencia es positiva el nuevo individuo reemplaza al anterior. En cambio, si la diferencia es negativa indica un aumento en el fitness, entonces se calcula el valor absoluto de ambas diferencias y se divide la diferencia del objetivo actual con el anterior. $\mathrm{Si}$ este cociente es menor que $\theta$ el nuevo individuo es aceptado, si no, se descarta.

\section{Diseño de experimentos}

Esta sección detalla cómo se llevaron a cabo los experimentos realizados en esta investigación, desde cómo se dividen las instancias de prueba utilizadas hasta el equipo donde se ejecutaron los algoritmos.

\section{Agrupación de las instancias de prueba}

Para el desafío de ROADEF en 2005 se utilizaron 3 conjuntos de instancias provenientes de diferentes fábricas de RENAULT. El primero, denominado conjunto $\mathrm{A}$, consta de 16 instancias que se utilizaron en la etapa de clasificación del desafío. 
El conjunto B está constituido de 45 instancias que fueron dadas a los participantes que superaron la primera etapa. El tercero, set X, se compone de 19 instancias que sirvieron como evaluación final del desafío. En este trabajo, se utilizan las instancias del conjunto A. La Tabla 1 muestra cómo están agrupadas las 16 instancias utilizadas para esta investigación.

\begin{tabular}{|c|c|c|}
\hline Grupo & Prioridad de los objetivos & $\begin{array}{c}\text { Nombres de } \\
\text { las } \\
\text { instancias }\end{array}$ \\
\hline Fáciles & $\begin{array}{l}\text { Estaciones de alta prioridad } \\
\text { (FH) } \\
\text { Cambios de color (FC) } \\
\text { Estaciones de baja prioridad } \\
\text { (FL) }\end{array}$ & $\begin{array}{l}\text { HEPL1, } \\
\text { HEPL2, } \\
\text { HEPL3, } \\
\text { HEPL4, } \\
\text { HELP }\end{array}$ \\
\hline Color & $\begin{array}{l}\text { Cambios de color (FC) } \\
\text { Estaciones de alta prioridad } \\
\text { (FH) } \\
\text { Estaciones de baja prioridad } \\
\text { (FL) }\end{array}$ & $\begin{array}{l}\text { PHEL1, } \\
\text { PHEL2, } \\
\text { PHEL3, PHE }\end{array}$ \\
\hline Difíciles & $\begin{array}{l}\text { Estaciones de alta prioridad } \\
\text { (FH) } \\
\text { Cambios de color (FC) } \\
\text { Estaciones de baja prioridad } \\
\text { (FL) }\end{array}$ & \begin{tabular}{|l|} 
HDLP1, \\
HDLP2, \\
HDLP3, \\
HDPL1, \\
HDPL2, \\
HDPL3, HDP \\
\end{tabular} \\
\hline
\end{tabular}

Tabla 1 Clasificación de las 16 instancias del conjunto A Fuente: elaboración propia [Microsoft Word]

En el primer grupo se encuentran las instancias que tienen como objetivo principal disminuir las sobrecargas de trabajo en las estaciones de alta prioridad, estas instancias son fáciles de resolver para RENAULT. El segundo grupo se compone de las instancias cuyo objetivo primordial es minimizar los cambios de color. Las instancias que priorizan el suavizado de sobrecargas en estaciones de alta prioridad y que son etiquetadas como difíciles de resolver pertenecen al grupo tres. En cualquier grupo, los objetivos 2 y 3 pueden variar en orden y en algunas instancias no se contemplan estaciones de trabajo de baja prioridad por lo que solo hay dos objetivos a optimizar (i.e. PHE y HDP).

Se realizaron 10 ejecuciones por algoritmo en cada una de las instancias pertenecientes al conjunto A utilizadas en el desafío de 2005. Esto debido a las especificaciones proporcionadas a los equipos participantes por la compañía Renault (Nguyen). Estas pruebas se realizaron en una PC GameFactor con procesador Intel Core i5-8400 y memoria RAM de 16 gb. Los algoritmos fueron programados en Java con el IDE Apache NetBeans v11.1.

\section{Resultados y Análisis}

Derivado de los experimentos realizados, la Tabla 2 muestra el número total de mejores resultados que obtuvieron los algoritmos. Estos resultados están separados por objetivo. Como se puede observar en la tabla, de las tres versiones de MEDAL y BUMDA el algoritmo con mejores resultados en los objetivos de $\mathrm{FH}$ y FC es MEDAL CT. Mientras que para el objetivo de FL, el mejor algoritmo es MEDAL ST. Un factor importante en la obtención de estos resultados puede ser la aleatoriedad implicada en el proceso de generación de nuevos individuos utilizada por MEDAL como se explica en la sección 3.2.

\begin{tabular}{|l|r|r|r|}
\hline \multicolumn{1}{|c|}{ Algoritmo } & \multicolumn{1}{c|}{ FH } & \multicolumn{1}{c|}{ FC } & FL \\
\hline MEDAL ST & 8 & 4 & 9 \\
\hline MEDAL CT & 9 & 9 & 2 \\
\hline BUMDA & 0 & 4 & 2 \\
\hline
\end{tabular}

Tabla 2 Total de mejores resultados obtenidos por los algoritmos

Fuente: elaboración propia [Microsoft Word]

Cabe señalar que en algunas instancias algunos algoritmos obtuvieron los mismos resultados, por eso en FH y FC la suma de las columnas difiere de 16. En el caso de FL, de las 16 instancias, 2 no contemplan este objetivo.

Para corroborar lo descrito en la Tabla 2 y las observaciones presentadas, se ejecutaron pruebas estadísticas no paramétricas (concretamente el Test de Friedman). En la siguiente subsección se detallan los resultados de esta prueba.

\section{Análisis de los resultados del Test de Friedman}

Se realizó el test de Friedman cuya hipótesis nula declara que no existe diferencia significativa en el comportamiento de los algoritmos. El nivel de significancia utilizado para esta prueba es de $0.05 \%$. Los valores $\mathrm{p}$ arrojados por la prueba son: para $\mathrm{FH} \mathrm{p}=0.000$ para $\mathrm{FC} \mathrm{p}=0.001$ y para FL $\mathrm{p}=0.000$, estos valores indican que sí se encuentra diferencia significativa entre los resultados arrojados por los algoritmos. En la Tabla 3 se muestran los rangos asignados en el test de Friedman. 


\begin{tabular}{|l|c|c|c|}
\hline \multicolumn{1}{|c|}{ Algoritmo } & FH & FC & FL \\
\hline MEDAL ST & 1.56 & 2.03 & 1.71 \\
\hline MEDAL CT & 1.50 & 1.72 & 1.89 \\
\hline BUMDA & 3.31 & 3.25 & 3.50 \\
\hline
\end{tabular}

Tabla 3 Rangos obtenidos por los algoritmos implementados

Fuente: elaboración propia [Microsoft Word]

En la tabla anterior se puede observar que, para los objetivos FH y FC, la versión de MEDAL que aplica tolerancia (MEDAL CT) en el proceso de sustitución de individuos obtuvo los mejores rangos. Para el objetivo FL, el algoritmo mejor posicionado es la versión de MEDAL que no usa tolerancia (MEDAL ST). Lo que confirma los datos mostrados en la Tabla 2. Se realizó la comparación de los dos mejores algoritmos (MEDAL CT y MEDAL ST) contra los datos proporcionados por Renault. Además, se realizó el test de Friedman con un nivel de significancia de $0.05 \%$. Los valores $p$ arrojados por la prueba son de 0.001 para el objetivo de $\mathrm{FH}$ y de 0.000 para los objetivos de FC y FL.

\begin{tabular}{|c|c|c|c|}
\hline \multicolumn{1}{|c|}{ Algoritmo } & FH & FC & FL \\
\hline RENAULT & 1.25 & 1.00 & 1.14 \\
\hline MEDAL ST & 2.41 & 2.66 & 2.29 \\
\hline MEDAL CT & 2.34 & 2.34 & 2.57 \\
\hline
\end{tabular}

Tabla 4 Rangos asignados por el Test de Friedman para los mejores algoritmos y los resultados de Renault Fuente: elaboración propia [Microsoft Word]

Como se muestra en la Tabla 4, los rangos de Renault son inferiores a los obtenidos por MEDAL CT y MEDAL ST. Destaca el hecho que, en FC, Renault obtiene un rango promedio de 1. Esto indica que Renault obtuvo los mejores resultados en las 16 instancias. Para los objetivos FH y FL el rango promedio de Renault es mayor a 1 , lo que indica que en algunas instancias los dos mejores algoritmos implementados en este trabajo obtuvieron mejores resultados que Renault.

\begin{tabular}{|l|r|r|r|}
\hline Instancia & \multicolumn{1}{c}{$\begin{array}{c}\text { Renault } \\
\text { FH }\end{array}$} & \multicolumn{1}{c|}{$\begin{array}{c}\text { MEDAL ST } \\
\text { FH }\end{array}$} & \multicolumn{1}{c|}{$\begin{array}{c}\text { MEDAL } \\
\text { CT FH }\end{array}$} \\
\hline PHEL1 & 197 & 81.5 & 97.5 \\
\hline PHEL3 & 462 & 350 & 350 \\
\hline
\end{tabular}

Tabla 5 Instancias donde se superaron los resultados de Renault en FH

Fuente: elaboración propia [Microsoft Word]
La Tabla 5 y la Tabla 6 muestran las instancias donde los algoritmos MEDAL CT y MEDAL ST obtienen mejores resultados que Renault. Se observa que para FH se logra disminuir la cantidad de sobrecargas en poco más de 100 unidades mientras que en FL se logran disminuir las sobrecargas hasta en 60 unidades. Este comportamiento muestra buenos indicios para seguir utilizando este tipo de algoritmos en este problema.

\begin{tabular}{|l|r|r|r|}
\hline Instancia & $\begin{array}{c}\text { Renault } \\
\text { FL }\end{array}$ & $\begin{array}{c}\text { MEDAL ST } \\
\text { FL }\end{array}$ & \multicolumn{2}{c|}{$\begin{array}{c}\text { MEDAL CT } \\
\text { FL }\end{array}$} \\
\hline PHEL3 & 883 & 826 & 821 \\
\hline
\end{tabular}

Tabla 6 Instancias donde se superaron los resultados de Renault en FL

Fuente: elaboración propia [Microsoft Word]

Adicional a los resultados estadísticos que se obtuvieron, un aspecto muy importante que se observó en la investigación es que los objetivos de suavizado y de color son altamente conflictivos ya que mientras el objetivo de suavizado tiende a crear conjuntos pequeños de autos (de tamaño q que depende de la restricción de capacidad de la estación evaluada), el objetivo de pintura requiere que se formen grupos grandes del tamaño de lote establecido en la instancia.

Para ilustrar lo anterior, en la Figura 6 se presenta un ejemplo en tres tablas (a), (b), (c). Cada tabla cuenta con tres renglones, el primer renglón es una secuencia de autos, el segundo renglón la necesidad de instalación o no de un aire acondicionado en el auto $(\mathrm{r}=1 \quad \mathrm{o} \quad \mathrm{r}=0$ respectivamente) y un tercer renglón con el número de color con que se pintará cada auto. En cada secuencia los números 530, 531 y 532 representan los números de autos no atendidos en la secuencia del dia anterior, el resto de los números de la secuencia son el orden de los autos a procesar el día actual. En el ejemplo se maneja como restricción del objetivo de color, que el número máximo de autos secuenciados del mismo color es 5 . Como restricción de capacidad de la estación, el que se pueden recibir tres autos, pero solo a uno se le puede instalar aire acondicionado. En cada tabla, en el segundo renglón, se pueden obtener las sobrecargas o conflictos de suavizado sumando el número de veces que se excede en 1 la solicitud de instalación de aire acondicionado al deslizar una ventana de tamaño de tres posiciones consecutivas a lo largo de la secuencia. 
Por otra parte, en cada tabla en el tercer renglón se pueden obtener los cambios de color contando las veces que se cambia de número del color al seguir la secuencia. En el ejemplo se puede observar la naturaleza conflictiva entre los objetivos de suavizado y color. Se parte de una secuencia mostrada en la tabla (a) que tiene el mismo número de sobrecargas y cambios de color, 5 en este caso, de la cual se obtienen las secuencias mostradas en las tablas (b) y (c).

La segunda secuencia (b) minimiza los cambios de color colocando consecutivamente los autos que tienen el mismo color teniendo un total de 2 cambios de color mientras que las sobrecargas suman 8. En la última secuencia (c) los autos han sido colocados para minimizar el total de sobrecargas en la estación de trabajo resultando en 4 sobrecargas, sin embargo, el total de cambios de color aumenta a 8 .

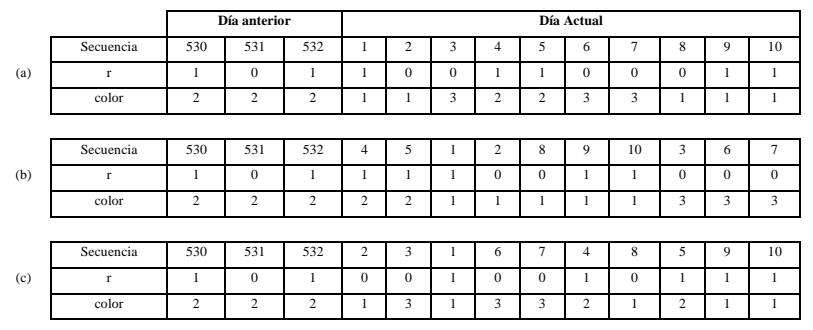

Figura 6 Conflicto entre objetivos, representado con el conteo de sobrecargas y cambios de color

Fuente: Elaboración propia [Microsoft Word]

\section{Conclusiones y trabajo a futuro}

En este trabajo se presentaron dos EDAs aplicados al problema de secuenciación de vehículos, la forma en que fueron adaptados y los resultados obtenidos. Se realizó un análisis estadístico para comparar el desempeño de MEDAL (en dos versiones) y BUMDA. Derivado de este análisis se concluye que la versión MEDAL CT obtuvo mejores soluciones para los objetivos FH y FC, mientras que para FL los mejores resultados fueron los de MEDAL ST. Para contrastar el desempeño de estas adaptaciones de EDAs con el estado del arte, estos algoritmos fueron comparados con los resultados de Renault.

Aunque las adaptaciones hechas al algoritmo MEDAL, no muestran competitividad con los resultados del estado del arte, si muestran la factibilidad para ser aplicado al CSP, la investigación permitió observar que se pueden mejorar los resultados evitando el conflicto contradictorio de los objetivos que afectaron el desempeño de los algoritmos.
Para mejorar el desempeño de los algoritmos y el conflicto contradictorio de éstos, se plantea dar continuidad a este trabajo incluyendo dos estrategias. Una relacionada con el estudio de los pesos adecuados a los objetivos en el enfoque lexicográfico y otra en la generación de clases en la secuenciación de vehículos.

\section{Agradecimientos}

Los autores agradecen al Consejo Nacional de Ciencia y Tecnología por el apoyo obtenido a través de la beca para estudios de posgrado: 930308 (Juan Carlos Velázquez Juárez), al Tecnológico Nacional de México/Instituto Tecnológico de León, a la Universidad de Guanajuato/División de Ciencias e Ingenierías Campus León y al SICES por el apoyo al proyecto CONV-PNPC/191/2019 por el apoyo brindado para la realización de esta investigación.

\section{Referencias}

Al Chami, Z., Manier, H., \& Manier, M. A. (2019). A lexicographic approach for the biobjective selective pickup and delivery problem with time windows and paired demands. Annals of Operations Research, 273 (1-2), 237-255. doi:https://doi.org/10.1007/s10479-017-2500-9

Armazañas, R., Saeys, Y., Inza, I., GarciaTorres, M., Bielza, C., van de Peer, Y., \& Larrañaga, P. (2011). Peakbin selection in mass spectrometry data using a consensus approach with estimation of distribution algorithms. IEEE/ACM Transactions on Computational Biology and Bioinformatics, 8 (3), 760-774.

Bacardit, J., Stout, M., Hirst, J. D., Sastry, K., Llorà, X., \& Krasnogor, N. (7-11 de Julio de 2007). Automated alphabet reduction method with evolutionary algorithms for protein structure prediction. (D. Thierens, Ed.) GECCO 2007: Genetic and Evolutionary Computation Conference, 346. doi:https://doi.org/10.1145/1276958.1277033

Ceberio, J., Irurozki, E., Mendiburu, A., \& Lozano, J. A. (2012). A review on estimation of distribution algorithms in permutation-based combinatorial optimization problems. Progress in Artificial Intelligence, 1(1), 103-117. doi:https://doi.org/10.1007/s13748-011-0005-3

VELÁZQUEZ-JUÁREZ, Juan Carlos, PUGA-SOBERANES, Héctor José, PADIERNA-GARCÍA, Luis Carlos y SÁNCHEZ-MÁRQUEZ, Elvi Malintzin. Estudio de factibilidad para la aplicación de algoritmos de estimación de distribución al problema de secuenciación de vehículos. Revista de Ingeniería Industrial. 2020 
Chutima, P., \& Olarnviwatchai, S. (2018). A multi-objective car sequencing problem on twosided assembly lines. Journal of Intelligent Manufacturing, 29(7), 1617-1636.

Duarte-Carrera, D., Rojas-Domínguez, A., \& Padierna García, L. (2018). Phase Unwrapping for 3D Object Reconstruction by means of Population-based Metaheuristics. Research in Computing Science, 147(11), 21-30.

Estellon, B., \& Gardi, F. (2013). Car sequencing is NP-hard: a short proof. Journal of the Operational Research Society, 64(10), 15031504. doi:https://doi.org/10.1057/jors.2011.165

Fliedner, M., \& Boysen, N. (2008). Solving the car sequencing problem via Branch \& Bound. European Journal of Operational Research, 191(3), 1023-1042. doi:https://doi.org/10.1016/j.ejor.2007.04.045

Gaudín, A., Madruga, G., \& Rodríguez, C. (2020). Planificación de vuelo utilizando algoritmos evolutivos. Informe de Proyecto de Grado, Universidad de la República Uruguay, Instituto de Computación - Facultad de Ingeniería, Montevideo, Uruguay.

Hauschild, M., \& Pelikan, M. (2011). An introduction and survey of estimation of distribution algorithms. Swarm and Evolutionary Computation, 1(3), 111-128. doi:https://doi.org/10.1016/j.swevo.2011.08.00 3

Ian P., G. (1998). Two Results on Carsequencing Problems. University of Strathclyde. (APES-02-98).

Jahren, E., \& Achá, R. A. (2018). A column generation approach and new bounds for the car sequencing problem. Annals of Operations Research, 264(1-2), 193-211. doi:https://doi.org/10.1007/s10479-017-2663-4

Kis, T. (2004). On the complexity of the car sequencing problem. Operations Research Letters, 32(4), 331-335. doi:https://doi.org/10.1016/j.orl.2003.09.003
Larrañaga, P., \& Lozano, J. A. (2002). Estimation of Distribution Algorithms: A New Tool for Evolutionary Computation. En Genetic Algorithms and Evolutionary Computation (Vol. 2, págs. 1568-2587). Springer US. doi:https://doi.org/10.1007/978-1-4615-1539-5

Nguyen, A. (s.f.). Challenge ROADEF' 2005 Car Sequencing Problem. RENAULT.

Palacio, J. D., \& Larrea, O. L. (2017). A lexicographic approach to the robust resourceconstrained project scheduling problem. International Transactions in Operational Research, 24(1-2), 143-157. doi:https://doi.org/10.1111/itor.12301

Parrello, B. D., Kabat, W. C., \& Wos, L. (1986). Job-shop scheduling using automated reasoning: A case study of the car-sequencing problem. Journal of Automated Reasoning, 2, 1-42.

Sánchez Márquez, E. M., Puga Soberanes, H. J., Mancilla Espinoza, L. E., Carpio Valadez, J. M., Ornelas Rodríguez, M., \& Manzanares Cuadro, J. I. (2019). Algoritmo de evolución diferencial con reparador cromosómico aplicado a un problema de secuenciación de vehículos. Research in Computing Science, 148(8), 279292.

Solnon, C., Cung, V., Nguyen, A., \& Artigues, C. (2008). The car sequencing problem: Overview of state-of-the-art methods and industrial case-study of the ROADEF'2005 challenge problem. European Journal of Operational Research, 191, 912-927.

Sun, J., Zhang, Q., Li, J., \& Yao, X. (2008). A Hybrid Estimation of Distribution Algorithm for CDMA Cellular System Design. International Journal of Computational Intelligence and Applications, $\quad 7(2), \quad$ 187-200. doi:https://doi.org/10.1142/S146902680800223 5

Valdez-Peña, S. I., Hernández, A., \& Botello, S. (2013). A Boltzmann based estimation of distribution algorithm. Information Sciences, 236 , 126-137. doi:https://doi.org/10.1016/j.ins.2013.02.040 